\title{
Estimating the Economic Burden of Rheumatoid Arthritis in Taiwan Using the National Health Insurance Database
}

\author{
Bruce C. M. Wang ${ }^{1}$ - Ping-Ning Hsu' ${ }^{2}$ Wesley Furnback ${ }^{1}$ • \\ John $\mathrm{Ney}^{3} \cdot$ Ya-Wen Yang ${ }^{4}$ Chi-Hui Fang ${ }^{4}$ - Chao-Hsiun Tang ${ }^{5}$
}

Published online: 16 March 2016

(c) The Author(s) 2016. This article is published with open access at Springerlink.com

\begin{abstract}
Background Rheumatoid arthritis (RA) is a chronic autoimmune disease characterized by inflammation and destruction of the joints.

Objectives This research aims to estimate the economic burden of RA in Taiwan.

Methods The National Health Insurance Research Database (NHIRD), a claims-based dataset encompassing $99 \%$ of Taiwan's population, was applied. We used a microcosting approach for direct healthcare costs and indirect social costs by estimating the quantities and prices of cost categories. Direct costs included surgeries, hospitalizations, medical devices and materials, laboratory tests, and drugs. The costs and quantities of the direct economic burden were calculated based on 2011 data of NHIRD. We identified RA patients and a control cohort matched 1:4 on demographic and clinical covariates to calculate the incremental cost related to RA. Indirect costs were
\end{abstract}

Electronic supplementary material The online version of this article (doi:10.1007/s40801-016-0063-8) contains supplementary material, which is available to authorized users.

Chao-Hsiun Tang

bruce.wang@elysiagroup.com; chtang@tmu.edu.tw

1 Elysia Group Ltd., Floor 2, No. 17-1, Lane 113, Xiamen Street, Taipei, Taiwan

2 National Taiwan University, No. 1, Changde St., Zhongzheng Dist., Taipei 10048, Taiwan

3 University of Washington, 1410 NE Campus Parkway, Seattle, WA 98195, USA

4 Pfizer Limited, 177 Chung Cheng East Road, sec. 2, Tansui, New Taipei City 251, Taiwan

5 Taipei Medical University, 250 Wuxing Street, Taipei 110, Taiwan evaluated by missed work (absenteeism) and worker productivity (presenteeism). For the indirect burden, we estimated the rate of absenteeism and presenteeism from a patient survey. Costs were presented in US dollars (US\$1 = 30 TWD).

Results A total of 41,269 RA patients were included in the database with incremental total direct cost of US\$86,413,971 and indirect cost of US\$138,492,987. This resulted in an average incremental direct cost of US\$2050 per RA patient. Within direct costs, the largest burdens were associated with drugs (US\$73,028,944), laboratory tests (US\$6,132,395), and hospitalizations (US $\$ 3,208,559$ ). For indirect costs, absenteeism costs and presenteeism costs were US $\$ 16,059,681$ and US\$114,291,687, respectively.

Conclusions The economic burden of RA in Taiwan is driven by indirect healthcare costs, most notably presenteeism.

\section{Key Points}

The average incremental direct cost of RA was US\$2050 per patient.

The economic burden of RA in Taiwan is driven by indirect healthcare costs, most notably, presenteeism.

\section{Introduction}

Rheumatoid arthritis (RA) is a chronic inflammatory disease of the joint synovium [1], leading to progressive disability and loss of function. RA is associated with 
cardiovascular diseases and premature death, but in working-age adults the symptoms of RA can result in diminished productivity, work absences, and ultimately unemployment [2]. RA exacerbations may require emergency department visits and inpatient hospitalization. With advances in RA treatment, many of these consequences can be avoided, albeit at some expense [3].

The costs of illness attributable to RA in Western nations has been well characterized. Direct healthcare costs and indirect costs from the effect of illness on the workforce shape estimates of the average and collective economic burden of RA [4]. Population-based studies from registries, administrative claims, and micro-costing studies have been instrumental in showing the societal burden of RA [5-7]. However, RA is a pervasive illness not limited to Western countries. RA has a global prevalence of $0.24 \%$, ranking 42nd out of 291 diseases in ascending contributions to global disability [8].

Taiwan is an attractive candidate for calculating the economic cost of illness for RA in a non-Western country because this nation has an educated population with an advanced healthcare system and a near complete insurance claims dataset [9]. A prior analysis using the National Health Insurance Research Database (NHIRD) calculated the prevalence of RA in Taiwan at 97.5/100,000 [10]. Therefore, our aim was to estimate the direct healthcare costs of RA in Taiwan using the NHIRD and calculate indirect costs due to absenteeism and lost productivity from a sample of working-age RA sufferers. The combined figure will be an estimate of the total economic burden of $\mathrm{RA}$ in Taiwan.

\section{Methods}

\subsection{Data Sources}

For the calculation of direct healthcare costs, we utilized cross-sectional data from Taiwan's NHIRD [11]. The National Health Insurance program of Taiwan provides mandatory universal healthcare coverage for Taiwanese citizens. The NHIRD is an administrative claims dataset which captures $99 \%$ of all medical claims for the Taiwanese citizens. Baseline demographics and healthcare utilization events are compiled annually, including diagnostic coding for medical conditions, as well as details and expenditures for prescriptions, medical devices, and inpatient and ambulatory care claims. These features have made the NHIRD an attractive source for population-based healthcare studies [12-15]. We used the following data subsets in our analysis: registry for catastrophic illness patients (HV), registry for beneficiaries (ID), inpatient expenditures by admissions (DD), details of inpatient orders (DO), ambulatory care expenditures by visits (CD), and details of ambulatory care orders (OO).

We determined RA exposure through the use of International Classification of Disease, Ninth Revision, Clinical Modification (ICD-9-CM) code [16] 714.xx (excluding 714.3) in the catastrophic illness file (a registry file that records all prevalent cases of patients who have major illnesses) of the NHIRD for rheumatoid arthritis associated with any healthcare event occurring during the 2011 calendar year. Patients who have catastrophic illnesses are exempt from co-payment. All prevalent RA patients in 2011 were included in the 2011 catastrophic illness file, so we captured all of the RA patients.

For indirect healthcare costs, we developed a patient questionnaire [Work Productivity and Activity Impairment Questionnaire: Rheumatoid arthritis (WPAI:RA)] [17-19] to determine missed work and reduced productivity while at work. The questionnaire was administered to RA sufferers aged 20-70 years identified from specialty care clinics. The survey was designed to over-sample patients with severe RA to ensure an adequate sample size for parameter estimation by level of severity. From June 2013 to May 2014, we conducted face-to-face patient interviews with patients aged between 20 and 70 years at the rheumatology outpatient clinics of four hospitals located in northern, central, and southern Taiwan after being approved by the ethics committee of each participating hospital. The sampling took a purposive procedure and no randomized procedure was used. In order to obtain the minimum acceptable number of patients for each level of RA severity, each physician was required to include at least ten patients per level of severity defined by Disease Activity Score (DAS 28).

2011 Taiwanese dollar to US dollar exchange rates were retrieved from Google Finance [20].

\subsection{Matching Criteria}

We matched these cases to non-RA sufferers (at random and with replacement for each RA case) at a ratio of 1:4 on the basis of demographics (age, gender, and level of urbanization) for all adults covered by the National Health Insurance program (see Supplementary Electronic Material Table 1, for patient numbers). Note that the 1:4 ratio is the most commonly used ratio by researchers when matching cases and controls because "there is usually no marginal increase in precision from increasing the ratio of controls to cases beyond four" [21]. Since the study took a casecomparison approach, issues such as multiple chronic conditions in patients occurred in both the cases and comparison groups. The differences in the utilization, e.g. medication, show the incremental medication costs between the two groups under their respective profiles of chronic conditions. 


\subsection{Microcosting}

The estimated economic burden of RA in Taiwan is the sum of the direct healthcare costs attributable to consequences and care for RA and the societal impact of RA on missed work (absenteeism) and worker productivity (presenteeism). To calculate the direct costs for each of the RA and non-RA cohorts, we took the total amount of healthcare events consumed by all patients and divided it by the total number of patients to calculate mean costs; there were no confidence intervals or standard deviation results generated in this process.

In line with previous studies [22, 23], we calculated the societal impact (indirect costs) of RA from the sum of annual missed work due to RA accounting for the loss of productivity for the time worked. Presenteeism was calculated by segmenting the patients into mild, moderate, or severe groups based on survey results. Based on the number of hours the patients reported being at work, their work productivity effect was analyzed. A work productivity effect of 10 (scale 1-10) was penalized as no work being done and a $100 \%$ forfeiture of wages during that time. A 5 was assessed as a $50 \%$ reduction of productivity and a $50 \%$ reduction in wages during that time was assessed.

The total burden of illness (BOI) for the nation of Taiwan is the product of the mean (incremental direct and indirect) costs associated with RA multiplied by the prevalent RA population (Fig. 1).

\subsection{Model Inputs}

Direct healthcare events of interest extracted from the 2011 NHIRD included inpatient events, ambulatory care events, and RA-related prescription pharmaceuticals. Inpatient events were decomposed into ICU bed stays, chronic bed stays, acute stays, and top ten surgical procedures. The top ten outpatient medical devices and diagnostic procedures used were sorted and used to inform the model. RA- relevant medications included biologic disease-modifying antirheumatic drugs (DMARDs), non-biologic DMARDs, non-steroidal anti-inflammatory drugs (NSAIDs), and steroids. The average costs for each of these parameters were also taken from the NHIRD 2011 dataset (See Table 1 for details of direct cost parameters).

Indirect cost inputs (from RA patient questionnaires) were rate of employment for RA patients, time lost due to RA, the effect of RA on productivity, and mean salary (Table 2).

\section{Results}

A total of 41,269 Taiwan National Health Insurance enrollees had healthcare events coding for RA in 2011. The mean age of the exposed cohort was 59.4 (range 2-105), $78.1 \%$ were female. Seventy-four percent of RA sufferers in the dataset were found in urban areas.

Under the constraint of time and costs, 140 RA sufferers of all severity levels (57 mild, 44 moderate, 39 severe) responded to the Work Productivity and Activity Impairment questionnaire for calculation of indirect costs [19]. Of these, $39.3 \%$ were employed, with unemployment, lost working hours, and impairment while working greater for more severe illness than for mild or moderate RA [24, 25].

The mean incremental direct costs attributable to RA were US\$2050 per person. The mean indirect costs per person were US $\$ 3356$. The economic burden of illness of RA for the nation of Taiwan in 2011 was US $\$ 224,906,868$.

The incremental direct healthcare costs of RA comprised US\$86,413,971 in 2011. The largest expenditures within direct healthcare costs were for RA-related prescription medications, amounting to US $\$ 73,028,944$, of which $67.4 \%$ were due to biologic DMARDs. Laboratory tests (US\$6,132,395) and inpatient ward costs (US\$3,208,559) were the next largest expenses.

Indirect costs were estimated at US $\$ 138,492,897$ (61.6\% of the total economic burden of illness). Although



Fig. 1 Calculating the total incremental burden (direct and indirect costs) associated with rheumatoid arthritis (RA) by differencing the costs of the RA cohort with the non-RA cohort matched 1:4 on age, gender, and level of urbanization 
Table 1 Estimated direct medical costs (in US\$ per patient) using the 2011 National Health Insurance Research Database of Taiwan for the rheumatoid arthritis (RA) patient cohort and the matched, non-RA cohort

\begin{tabular}{|c|c|c|c|}
\hline \multirow[t]{2}{*}{ Medications } & \multicolumn{2}{|c|}{ Per patient } & \multirow{2}{*}{$\begin{array}{l}\text { Incremental total } \\
\text { costs }\end{array}$} \\
\hline & $\begin{array}{l}\text { RA } \\
\text { group }\end{array}$ & $\begin{array}{l}\text { Control } \\
\text { group }\end{array}$ & \\
\hline \multicolumn{4}{|l|}{ Biologic DMARDs } \\
\hline Etanercept (25 mg) & 678.14 & 0.00 & $27,986,003$ \\
\hline Adalimumab (40 mg) & 389.30 & 0.78 & $16,033,710$ \\
\hline Rituximab (10 ml) & 0.61 & 0.31 & 12,662 \\
\hline Rituximab (50 ml) & 126.61 & 1.42 & $5,166,522$ \\
\hline \multicolumn{4}{|l|}{ Tablet DMARDs } \\
\hline Azathioprine & 6.56 & 0.26 & 260,032 \\
\hline Cyclosporin & 67.31 & 0.94 & $2,738,805$ \\
\hline Hydroxychloroquine & 50.58 & 0.30 & $2,075,102$ \\
\hline Leflunomide & 151.91 & 0.14 & $6,263,503$ \\
\hline Methotrexate & 9.74 & 0.01 & 401,220 \\
\hline Penicillamine & 0.93 & 0.01 & 38,081 \\
\hline Sulfasalazine & 54.69 & 0.13 & $2,251,840$ \\
\hline \multicolumn{4}{|l|}{ NSAIDs } \\
\hline Celecoxib & 163.37 & 3.83 & $6,584,097$ \\
\hline Etodolac & 4.09 & 0.45 & 150,359 \\
\hline Etoricoxib & 42.88 & 2.26 & $1,676,482$ \\
\hline Meloxicam & 24.41 & 1.71 & 936,907 \\
\hline Nabumetone & 0.90 & 0.12 & 32,324 \\
\hline Nimesulide & 0.63 & 0.10 & 22,212 \\
\hline \multicolumn{4}{|l|}{ Steroids } \\
\hline Dexamethasone (oral) & 0.06 & 0.02 & 1,496 \\
\hline Dexamethasone (injection) & 9.91 & 0.28 & 397,574 \\
\hline Prednisolone & 0.00 & 0.00 & 14 \\
\hline \multicolumn{4}{|l|}{ Surgery } \\
\hline Lens surgery & 29.22 & 19.50 & 401,279 \\
\hline Joint replacement surgery & 17.93 & 4.78 & 542,954 \\
\hline Neurosurgery & 17.14 & 7.34 & 404,162 \\
\hline Bone fracture surgery & 7.06 & 3.81 & 134,259 \\
\hline Revision joint replacement surgery & 6.68 & 2.38 & 177,767 \\
\hline $\begin{array}{l}\text { Extracorporeal shock wave lithotripsy for urinary } \\
\text { calculi }\end{array}$ & 4.17 & 3.57 & 24,498 \\
\hline Integumentary surgery & 3.95 & 2.40 & 64,332 \\
\hline Artery and vein surgery & 2.91 & 2.47 & 18,310 \\
\hline Heart and pericardiotomy surgery & 2.37 & 1.61 & 31,292 \\
\hline Intestine (excluding rectum) surgery & 2.19 & 1.67 & 21,633 \\
\hline \multicolumn{4}{|l|}{ Ward usage } \\
\hline ICU & 51.02 & 24.79 & $1,082,816$ \\
\hline Acute beds & 95.88 & 41.56 & $2,241,755$ \\
\hline Chronic beds & 5.48 & 8.29 & $-116,012$ \\
\hline \multicolumn{4}{|l|}{ Medical devices and materials } \\
\hline Artificial function substitutes for orthopedics & 52.78 & 12.93 & $1,644,683$ \\
\hline Catheters for balloon angioplasty & 8.64 & 5.04 & 148,562 \\
\hline Blood transfusion sets/fluid transfer sets & 5.00 & 2.28 & 112,214 \\
\hline Pressure monitoring kit & 3.76 & 1.48 & 94,281 \\
\hline Cardiovascular & 4.63 & 3.10 & 62,935 \\
\hline Drainage tube & 2.15 & 1.19 & 39,615 \\
\hline
\end{tabular}


Table 1 continued

\begin{tabular}{lccc}
\hline Medications & Per patient & & Incremental total costs \\
\cline { 2 - 3 } & RA group & Control group & \\
\hline Pilot tube & 2.44 & 1.61 & 34,367 \\
Special material management fees & 1.07 & 0.26 & 33,226 \\
Connecting tubes & 1.23 & 0.56 & 27,788 \\
Renal and ureteral catheters & 1.60 & 0.97 & 25,916 \\
Laboratory tests & & & \\
Immunology examination & 62.20 & 6.40 & $2,302,764$ \\
Hematology test & 34.40 & 7.07 & $1,128,024$ \\
Biochemistry examination & 48.75 & 23.47 & $1,043,384$ \\
Circulative function examination & 17.41 & 10.08 & 302,503 \\
Radioisotope scanning & 13.52 & 6.68 & 282,244 \\
Sonography & 14.01 & 7.41 & 272,179 \\
Specimen examination & 13.32 & 6.95 & 262,863 \\
Bacteriology and fungal test & 9.76 & 3.78 & 246,846 \\
Endoscopy examination & 9.81 & 5.71 & 168,901 \\
Neurological test & 6.48 & 3.51 & 122,686 \\
\hline
\end{tabular}

DMARD disease-modifying antirheumatic drug, NSAID nonsteroidal anti-inflammatory drug, ICU intensive care unit

Table 2 Estimated indirect, societal burden parameters from the WPAI:RA questionnaire stratified by rheumatoid arthritis (RA)

\begin{tabular}{llll}
\hline Input & Mild RA & Moderate RA & Severe RA \\
\hline Severity distribution & $42.11 \%$ & $32.63 \%$ & $25.26 \%$ \\
Number currently employed & $37.50 \%$ & $38.71 \%$ & $37.50 \%$ \\
Hours missed due to RA (per week) & 2.07 & 1.25 & 4.11 \\
Productivity loss & $27.30 \%$ & $38.30 \%$ & $67.80 \%$ \\
Average yearly salary [32] & US\$18,920 (NT\$567,600) & \\
\hline
\end{tabular}

WPAI:RA Work Productivity and Activity Impairment Questionnaire: Rheumatoid arthritis less than $40 \%$ of RA patients surveyed had full-time employment, missed work due to RA (less than 2 hours per employed worker per week) accounted for less than $15 \%$ of the total indirect costs. Impaired productivity in RA sufferers (mean loss of $41 \%$ of productivity) cost the economy of Taiwan US\$121,430,155 in 2011.

\section{Discussion}

Economic burden of illness models facilitate understanding of the shared costs to society for disabling health conditions [26]. Because illness is distributed differently in different countries, nation-specific data can further emphasize the variation in expenditures and lost labor where geographic, cultural, governmental, and economic factors differ. For Taiwan, the nearly complete dataset available for healthcare usage through the National Health Insurance program allows for accurate nation-level evaluation of attributable direct healthcare costs, while patientreports of effects on missed work and reduced productivity estimate the impact of RA on the labor force of Taiwan. Here, we note that the majority of direct healthcare costs are due to the expense of pharmaceutical treatments for RA, but this is outweighed by the indirect costs of RA, particularly on worker productivity.

Direct healthcare costs of RA are a function of utilization of healthcare resources. While only a minority of RA sufferers will use acute care in the form of emergency room visits and inpatient hospitalization in a given year, nearly all RA sufferers experience routine outpatient evaluation and treatment of the illness. The costs of treatment in particular are multiplied over nearly the entire prevalent RA population, and the prescription medications with the greatest expense per person (biologic DMARDs) are reflected in the percentage of direct healthcare costs for Taiwan (greater than $55 \%$ ).

Much of the disability of rheumatoid arthritis is inflicted by inflammatory damage from the illness on the joints of RA sufferers. In reducing the symptoms of joint inflammation from RA, the degree of disability can be ameliorated. The DMARDs, particularly the newer biological 
DMARDs, have shown particular efficacy in curbing RA where chronic inflammation causes permanent damage and disability.

The indirect costs of RA are due in large part to RArelated disability affecting job performance [4]. Pain and swelling in the joints of the hand and wrist are highly disruptive to both manufacturing and office work [27]. Taiwanese workers with RA, though having a relatively low rate of absenteeism, experience marked productivity losses that are attributable to their disease. Furthermore, as the severity of the illness increases, the degree of work impairment is greater.

The indirect economic costs depicted here, while representative of RA-related disability, likely understate the effect of RA on quality of life and function. The questionnaire respondents were not a representative sample of the RA population in Taiwan. Less than half of the questionnaire respondents were employed, with unemployment higher with increased disease severity. Absenteeism and presenteeism fail to capture the daily struggles in the $60 \%$ of persons who are not part of the Taiwanese work force. Impairment in activities of daily living including walking, shopping, cooking, and cleaning may be substantial in this group.

Relative to other conditions evaluated using Taiwan NHIRD data, costs for RA are large. In a matched, casecontrol study of Taiwanese citizens with chronic rhinosinusitis, the mean incremental direct healthcare costs were US\$373 for nearly 6000 persons [28]. An evaluation of the direct and indirect healthcare costs of psoriasis, an inflammatory skin condition with similar prescription treatments to RA, showed a total cost of US\$33.4 million annually (in 2009 dollars), distributed among over 51,000 prevalent persons [29]. Only $10 \%$ of costs were due to loss of productivity. In our study, we note a greater mean and total cost for RA than these conditions, and a far greater share of cost due to worker productivity loss than in psoriasis.

This analysis is comparable to burden-of-illness studies for RA in Western countries. A population-based analysis of indirect and direct costs attributable to RA in Sweden [5] showed the total national burden of illness of 600 million euros (for around 20,000 prevalent persons) in 2010, of which $58 \%$ were indirect costs and prescription drug costs were $33 \%$ of total costs. An economic model of the cost of illness of RA in Italy [6] concluded that the total annual cost was 3.5 billion euros for a prevalent population of over 250,000 persons with RA, where indirect and non-medical direct costs comprised $79 \%$ of the total. Estimates of tangible costs (direct and indirect) of RA in the USA (not due to premature mortality or loss of quality of life) have been estimated to be up to US\$19.2 billion [30], and prevalence estimated at 1.3 million adult Americans with
RA [31]. Our findings show that per-person annual costs of RA in Taiwan are greater than those in Sweden but substantially less than estimates for US and Italian RA sufferers.

There are several limitations to this study. First, we examined annual direct healthcare expenditures by looking at a single year of data. The effects of treatment on the course of a chronic illness, which can last for many years, were outside the scope of this study as they would require multi-year analyses. Second, we assumed that a convenience sample of RA sufferers from specialty clinics were representative of the prevalent RA population. While the age range for the population-based study (from the NHIRD) is greater (age 2-105 years), we limited the questionnaire respondents to those of working age (20-70 years) to gauge the effects of RA on the workforce. Third, the estimation of indirect costs was limited to the RA sufferer, and did not include caretaker costs, lost work, and other expenses incurred by family and friends of the RA patient. Fourth, we did not evaluate the counterfactual argument regarding potential contributions to the workforce that currently unemployed RA sufferers could make if they did not have RA. The net effect of these latter two criticisms is an underestimation of the true burden of the illness as pertains to indirect economic costs of RA. Lastly, since the NHIRD is a claims-based dataset, our calculations may be underestimating the actual cost of RA. For example, home-based or institution-based long-term care is not covered by the National Health Insurance.

\section{Conclusion}

The burden of illness of RA in Taiwan is substantial. The direct healthcare costs, although large, are overshadowed by the loss of productivity of workers with the condition. This creates an opportunity for improved function while at work and home to offset the cost of new and effective treatments for RA in Taiwan.

Acknowledgments This study was based in part on data from the Taiwan National Health Insurance Research Database provided by the Bureau of National Health Insurance, Department of Health and managed by National Health Research Institutes. The interpretation and conclusions contained herein do not represent those of Bureau of National Health Insurance, Department of Health, or National Health Research Institutes.

\section{Compliance with Ethical Standards}

Funding This study was funded by Pfizer Taiwan.

Conflict of interest Bruce Wang and Wesley Furnback are employees of the Elysia Group who were paid consultants to Pfizer in connection with the development of this study and manuscript. John Ney was a paid consultant to Pfizer in connection with the 
development of this study and manuscript. Ya-Wen Yang is an employee of Pfizer Taiwan. Chi-Hui Fang was an employee of Pfizer during the development of the study and manuscript. Chao-Hsiun Tang and Ping-Ning Hsu have no conflicts of interest that are directly relevant to the content of this study.

Ethical statement The study protocol (data algorithm and questionnaire) was approved in advance by the Institutional Review Board of the National Taiwan University Hospital. Patient-level observations used to calculate direct healthcare costs were stripped of personal identifiers prior to analysis, therefore written informed consent from the patients involved was not necessary.

Open Access This article is distributed under the terms of the Creative Commons Attribution-NonCommercial 4.0 International License (http://creativecommons.org/licenses/by-nc/4.0/), which permits any noncommercial use, distribution, and reproduction in any medium, provided you give appropriate credit to the original author(s) and the source, provide a link to the Creative Commons license, and indicate if changes were made.

\section{References}

1. Khan MA. Update on Spondyloarthropathies. Ann Intern Med. 2002;136:896-907.

2. Uhlig T, Moe RH, Kvien TK. The burden of disease in rheumatoid arthritis. Pharmacoeconomics. 2014;32(9):841-51.

3. Barnabe C, Thanh NX, Ohinmaa A, et al. Effect of remission definition on healthcare cost savings estimates for patients with rheumatoid arthritis treated with biologic therapies. J Rheumatol. 2014;41:1600-6.

4. Filipovic I, Walker D, Forster F, Curry AS. Quantifying the economic burden of productivity loss in rheumatoid arthritis. Rheumatology (Oxford). 2011;50:1083-90.

5. Kalkan A, Hallert E, Bernfort L, Husberg M, Carlsson P. Costs of rheumatoid arthritis during the period 1990-2010: a registerbased cost-of-illness study in Sweden. Rheumatology (Oxford). 2014;53:153-60.

6. Turchetti G, Bellelli S, Mosca M. The social cost of rheumatoid arthritis in Italy: the results of an estimation exercise. Reumatismo. 2013;65:271-7.

7. Birnbaum H, Pike C, Kaufman R, et al. Societal cost of rheumatoid arthritis patients in the US. Curr Med Res Opin. 2010;26:77-90.

8. Cross M, Smith E, Hoy D, Carmona L. The global burden of rheumatoid arthritis: estimates from the Global Burden of Disease 2010 study. Annal rheumatic diseases 2014;73:1316-22.

9. Hsiao F-Y, Yang C-L, Huang Y-T, Huang W-F. Using Taiwan's National Health Insurance research database for pharmacoepidemiology research. J Food Drug Anal. 2007;15:99-108.

10. Kuo CF, Luo SF, See LC, Chou IJ, Chang HC, Yu KH. Rheumatoid arthritis prevalence, incidence, and mortality rates: a nationwide population study in Taiwan. Rheumatol Int. 2013;33:355-60.

11. National Health Insurance Research Database, Taiwan. Available at: http://nhird.nhri.org.tw/en/index.htm. Accessed 26 Oct 2015.

12. Chen GL, Hsiao FY, Dong YH, Shen LJ, Wu FL. Statins and the risk of liver injury: a population-based case-control study. Pharmacoepidemiol Drug Saf. 2014;23(7):719-25.

13. Chiang FY, Lu IC, Kuo WR, Lee KW, Chang NC, Wu CW. The mechanism of recurrent laryngeal nerve injury during thyroid surgery-the application of intraoperative neuromonitoring. Surgery. 2008:743-9.
14. Huang WY, Wu SC, Chen YF, Lan CF, Hsieh JT, Huang KH. Surgeon volume for percutaneous nephrolithotomy is associated with medical costs and length of hospital stay: a nationwide population-based study in Taiwan. $\mathrm{J}$ Endourol. 2014;28(8):915-21. doi:10.1089/end.2014.0003.

15. Wang JY, Wang CY, Huang YS, et al. Increased risk of ischemic stroke after hyperosmolar hyperglycemic state: a populationbased follow-up study. PLoS One. 2014;9:e94155.

16. Classification of Diseases, Functioning, and Disability, International Classification of Diseases, Ninth Revision, Clinical Modification (ICD-9-CM). Center for Disease Control and Prevention, 2011. http://www.cdc.gov/nchs/icd/icd9cm.htm. Accessed 18 July 2011.

17. Reilly MC, Zbrozek AS, Dukes E. The validity and reproducibility of a work productivity and activity impairment measure. PharmacoEconomics. 1993;4(5):353-65.

18. Reilly MC, Gooch KL, Wong RL, Kupper H, van der Heijde D. Validity, reliability and responsiveness of the Work Productivity and Activity Impairment Questionnaire in ankylosing spondylitis. Rhuematology. 2010;49(4):812-9. doi:10.1093/rheumatology/ kep457.

19. Chaparro del Moral R, Rillo OL, Casalla L, et al. Work productivity in rheumatoid arthritis: relationship with clinical and radiological features. Arthritis. 2012;2012:7. doi:10.1155/2012/ 137635 (Article ID 137635).

20. Google Finance. US Dollar (\$)—New Taiwan Dollar (NT\$). https://www.google.com/finance?q=USDTWD. Accessed 16 Dec 2014.

21. Tang CH, Hsu PN, Tsai ST, Shen CY, Tsai WC, et al. Healthstate utilities in measuring health-related quality of life among patients with rheumatoid arthritis in Taiwan. A Poster presented at ISPOR European Congress held in Dublin, Ireland, 2-6 November 2013.

22. Wacholder S, Silverman DT, McLaughlin JK, Mandel JS. Selection of controls in case-control studies: III. Design options. Am J Epidemiol. 1992;135:1042-50.

23. Wang B, Tang CH, Furnback W, Ney J, Yang YW, Fang CH, Huang YH. Disease burden of ankylosing spondylitis in Taiwan: a population-based analysis. In: European league against rheumatism meeting, Rome, 2015.

24. Wang B, Tang CH, Furnback W, Ney J, Yang YA, Fang CH, Huang YH. Disease burden of psoriatic arthritis in Taiwan: a population-based analysis. In: International society for pharmacoeconomics and outcomes research international meeting, Philadelphia, 2015.

25. Hsu JY, Chuang PY, Lin YS, Hsu PN, Tang CH. Using health assessment questionnaire-disability index to estimate EQ-5D utility values for patients with rheumatoid arthritis in Taiwan. A poster presented at ISPOR European Congress held in Amsterdam, Netherland, 8-12 November 2014.

26. Gold MR, Siegel JE, Russell LB, Weinstein MC. Cost-effectiveness in health and medicine. New York: Oxford University Press; 1996.

27. Kleinman NL, Cifaldi MA, Smeeding JE, Shaw JW, Brook RA. Annual incremental health benefit costs and absenteeism among employees with and without rheumatoid arthritis. J Occup Environ Med. 2013;55:240-4.

28. Chung SD, Hung SH, Lin HC, Lin CC. Health care service utilization among patients with chronic rhinosinusitis: a populationbased study. Laryngoscope. 2014;124:1285-9.

29. Chen K-C, Hung S-T, Yang C-WW, Tsai T-F, Tang C-H. The economic burden of psoriatic diseases in Taiwan. J Dermatol Sci. 2014;75:183-9.

30. Birnbaum H, Pike C, Kaufman R, Marynchenko M, Kidolezi Y, Cifaldi M. Societal cost of rheumatoid arthritis patients in the US. Curr Med Res Opin. 2010;26(1):77-90. 
31. Helmick CG, Felson DT, Lawrence RC, et al. Estimates of the prevalence of arthritis and other rheumatic conditions in the United States: part I. Arthritis Rheum. 2008;58:15-25.

32. National Statistics. Republic of China (Taiwan). Earnings and Productivity Statistics in August 2015. October 22, 2015.
Available at: http://eng.stat.gov.tw/public/Data/5102284719 W7OZ7PW0.pdf. Accessed 26 Oct 2015. 